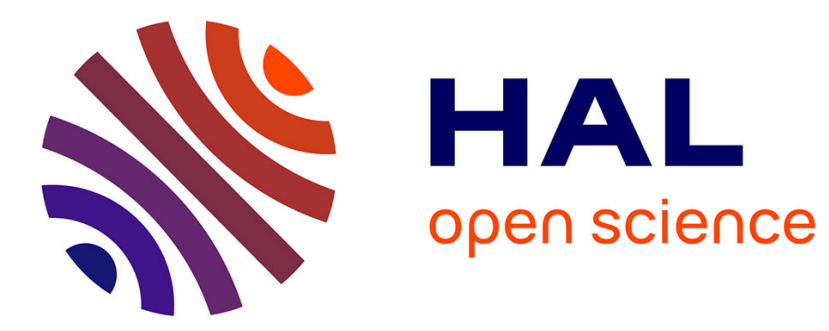

\title{
Can Organizations Really Unlearn?
}

Emil Turc, Philippe Baumard

\section{To cite this version:}

Emil Turc, Philippe Baumard. Can Organizations Really Unlearn?. Rethinking Knowledge Management, 2007. hal-01802249

\section{HAL Id: hal-01802249 \\ https://hal-amu.archives-ouvertes.fr/hal-01802249}

Submitted on 12 May 2021

HAL is a multi-disciplinary open access archive for the deposit and dissemination of scientific research documents, whether they are published or not. The documents may come from teaching and research institutions in France or abroad, or from public or private research centers.
L'archive ouverte pluridisciplinaire HAL, est destinée au dépôt et à la diffusion de documents scientifiques de niveau recherche, publiés ou non, émanant des établissements d'enseignement et de recherche français ou étrangers, des laboratoires publics ou privés. 


\title{
Can Organizations Really Unlearn?
}

\author{
Emil Turc ${ }^{1}$ and Philippe Baumard ${ }^{2}$ \\ ${ }^{1}$ Institute of Public Management, Université Paul Cézanne, Aix-en-Pce \\ ${ }^{2}$ Haas School of Business, University of gatifonnia, Berkeley
}

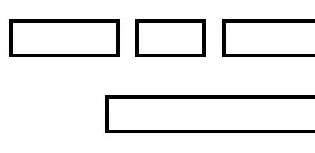

\begin{abstract}
The corbplex phentomedion of orddnizational change is a continuous challenge for scientists and for practitioners alike. Simple models tend to be regularly worn out by field evidence. More and more factors must be taken into account in order to ensure a better reliability of change models. A recently proposed solution suggests that learning theory should be placed more centrally within the theory of planned organizational change (Hendry, 1996; Schein, 1993. Kilmann, 1989). Hbwever, this nedearch diredtion has already been broached, although under a slightly different perspective. Early studies (Starbuck and Heberg, 1976; Hedberg et al. 1976) have shown that organizational change should initially go through an unlearning phase. The elimination of old, obsolete organizational knowledge - that is, unlearning - makes room for the development of new adaptive capacities (Hedberg, 1981; Nystrom and Starbuck, 1984; Hedberg et al. 1976; Markoczy, 1994; Starbuck, 1989).

This paper reviews the different conceptualizations of the unlearning process in the research literature. The integration of these various perspectives allows inferring that organizational unlearning is mainly apprehended as a tool for the removal of inefficient behavior in favor of an adaptive one. A subsequent analysis of the intimate bonds between organizationa_knowledge and actions (Pfeffer and Sutton, 1999: Kuwada, 1998; Klein, 1989) shows that ber knowledge maniplation processes
\end{abstract} may have the same behavioral effect. Two new processes are proposed. Knowledge inactivation and rivaling enforced enactment eliminate undesired behaviors by altering the perceived validity and, respectively, the operational capacity of underlying organizational knowledge. All together, unlearning, rivaling enactment, and knowledge inactivation are labeled as knowledge neutralization phenomena.

The article concludes over the place of the newly proposed class of processes in a change context. It is argued that, although the neutralization of old knowledge is not imperative for learning, its behavioral effects provide support for organizational change. Furthermore, knowledge inactivation, rivaling enactment, and unlearning seem to fit in specific organizational settings, according to the time and resources available. An analysis of management literature uncovers latent evidence for these findings (Lorsch, 1986; Starbuck and Laudon, 1996; Carmona and Grönlund, 1998). 


\section{Introduction}

Organizations face recurrent cognitive inertia. While they cope with unexpected and unwanted change, organizations may adapt through somatic change, but most of their knowledge lies unaffected Evdebtually, the very same knowledge that led them to previous success may turn out to be the very source of their next disaster Nystrom and Starbuck (1984) suggested that organizations should allocate part of their resources to "unlearning" in order to avoid further and recurrent oroanizational crises Tlittle is said however on how organizations may process in order to accomplish a durable and sustainable unlearning process. This paper investigates how organizations can really unlearn, and proposed a rivaling cognitive enactment process that may contribute to this achievement.

We investigate unlearning in a context of change. Previous research showed the interest of studying dynamic knowledge transformation at times of abrupt change or inextricable crises (Baumard, 1999). Our first assumption is that

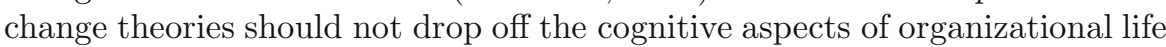
(after Meyer, 1982). Change and learning theories should be included in an integrative framework in order to draw a comprehensive image of processes

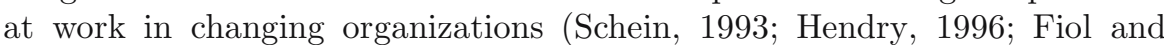
Lyles, 1985). Our second assumption is that dict otomous approaches might fail to notice important implications at the frontier of the two phenomena. Lorsch (1986) for instance sustained that neglecting culture, a learned group phenomenon, may cause serious impediments in strategic change. Presently held beliefs and methods shape perceptions, thus blinding people to potential interpretations of evidence. They also create strong emotional bonds resulting in strong attachment of organizational actors toward them (Hatch

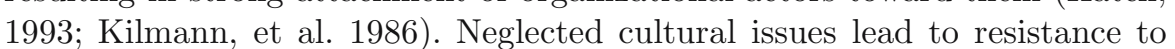
change, which results inhalf-acconnplished, flawed transformations. Certain management scientists grew to conceive organizational changes only through the lens of cultural revolutions (Firsird w, 1985; Kilmann, 1989).

Alternatively, Weick (1979) suggested that organizations couldn't foster new knowledge unless room is made for new ideas and cognitive frameworks. This new perspective introduced dialectic between old and new knowledge, and old and new learning, as an embedded core process of organizing. Old knowledge is, thus, perceived as an obstacle to renewed cognitive frameworks and new learning, and need to be discontinued before new knowledge can be generated. This process of discarding obsolete knowledge is called unlearning (Hedberg, 1981; Nystrom and Starbuck, 1984; Hedberg, et al. 1976; Markóczy, 1994; Starbuck, 1989).

Organizational knowledge doesn't determine performance; behavior does (Pfeffer and Sutton, 1999). Management research considers the unlearning of old knowledge mainly as a tool for the removal of inefficient behavior in favor of an adaptive one. It is arguable however that unlearning shall be the only option available to reach this goal. Adopting a cognitive perspective, this 
paper sets out to demonstrate that unlearning represents only a particular stance of a whole class of processes with similar effects. These processes, brought together under the label of knowledge neutralization, include phenomena such as knowledge invalidation and rivaling enforced enactment. We argue that making old knowledge lose its grip over organizational behavior has been the blind spot in the development of unlearning theories.

The first part of this paper re-examines previous conceptualizations of organizational unlearning, emphasizing the processes and the roles attributed to this phenomenon by management scientists. The second part discusses aspects insufficiently developed or ignored by unlearning theories, such as knowledge validity and activation. This review allows a broader conceptualization of the unlearning issue under the name of knowledge neutralization. The three types of processes identified thereof are then attentively examined and illustrated in the third part of the article. A final section discusses these findings with respect of turning unlearning as a durable and sustainable routine in organizations.

\section{Unlearning Thdories in the Wake of Organizational Learning: Roles and Processes}

For certain scientists, Organizational Learning (OL) is equivalent to a good adaptation of the organization thits environment through simple processes of habit formation. Subsequently, learning would merely be the retention of successful response patterns for reactive use. Recent developments considered a broader range of phenomena: organizations engage in such actions as exploration and experimentation (Nicolini and Meznar, 1995). They thus develop insights, enact their environments and subsequently memorize the causal relationships they had discovered (Hedberg, 1981; Weick, 1999). Organizations make an offensive yse of this knowledge in order to achieve a better fit with their enviddnments, and an enhancement of their effectiveness. Learning becomes "the process of developing a potential to improve actions (behavior) through better knowledge and understanding (cognition)" (Villinger, 1996).

Organizational knowledge represents the focal point of organizational learning. It develops from different experimental settings provided by the environment. However, knowledge grows and it simultaneously becomes obsolete as reality changes. Therefore, understanding involves both new learning and eliminating misleading and obsolete knowledge. The discarding activity has been called organizational unlearning (Hedberg, 1981).

One of the first researchers to address the unlearning phenomenon is William Starbuck (1989). According to this author's model of organizational crisis, firms do not only build knowledge by interaction with their environments. They also build trust in their old practices, beliefs, values and frames of reference. However, the repeated success of their application 
eventually blinds the organizational actors. They will no longer heed signals that are inconsistent with their old knowledge. When the organization's environment changes, disquieting information such as funds shortages, falling revenues, and actual losses, is accounted for as random deviations. The market must be adapting to a new entrant, the decline of applications must be due to accidental fluctuations, and prices willtakesombtimle to readjust because the currency was shaken. The firms enter a first phase of "weathering-the-storm": money is raised through budget trimming and the shedding of peripheral activities; controls are centralized.

At length, however, all slack resources are consumed and the company enters an "unlearning phase" while the incipient crisis turns into a fullblown one. The leaders issue inconsistent messages. People feel disoriented and low morale spreads throughout the firm. Managers who have long time reported that the hard times were ending, loose their credibility. In the end, the worldview and the standard operating procedures break down. The organization has unlearned its past and is now either heading to its end or is busily relearning (Starbuck, 1989).

According to the model of organizational crisis proposed by Starbuck, unlearning is an abrupt process that will precede new learning. Declining organizations don't have the time to change at their own pace: they must change and they must do so quickly. It is suggested that the quickest way to unlearn is to fire the top managers. Two aims are thus attained. First, managers represent a strategic part of the organizational "hardware" in which knowledge is recorded and they can translate this knowledge into action. Eliminating management thus means to eliminate important, active, parts of the organizationa_memory. Second, employees tend to associate ideas with their promoters, i.e their managers. Their departure is thus charged with a symbolic value. It will implicitly signify the and of the valitity of old values and beliefs and the necessity of seeking new ones. Either way, this viewpoint supposes, first, the deletion of old knowledge (unlearning), and second, the search for, and an experimentation with, new knowledge, which will come to replace the old. Unlearning is a distinctive part of the learning process: unlearning and relearning proceed sequentially. Unlearning also triggers the relearning process.

Besides eradicating complete physical parts of the organizational memory, scientists have also suggested an unlearning modality that supposes the cognitive elimination of knowledge from the organizational memory. Hedberg (1981), discussing the two-level model of organizational learning originally proposed by Argyris and Schön (1976), concludes that "knowledge that has resulted from complete learning cycles in organizations can normally be unlearned through complete cycles too." In other words, knowledge that has been learned through trial-and-error processes, can be rejected through the same trial-and-error cycles. Changing environments induce the unlearning of past knowledge that will consequently eliminate obsolete organizational behaviors. The unlearning process triggers the relearning 


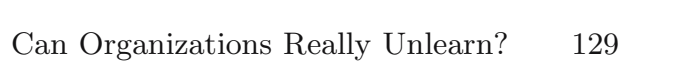

process that, through experimentation, imitation, or political dynamics will lead to the creation of new, suitable knowledge for the present environment. The outcome of the relearning process will be the appopriation of new adaptive organizational behaviors (Starbuck, 1989; Hedberg, et al. 1976).

\section{Unlearning: A Controversial Process}

Considering unlearning as a distinctive part of the learning process has sometimes been deemed artificial (Nicolini and Meznar, 1995). Learning and unlearning, when their distinction is meaningful, often flow concomitantly and not sequentially. Furthermore, this perspective leads to considering knowledge as a stock that can be mobilized or retrieved upon urgent needs, discarded at one's will. As Starbuck and Laudon (1996) pointed out, people in organizations preserve knowledge by applying it, and renew knowledge the very same way Thus, applying is both of sowles of preservation and elimination. How then can people know that they are learning a new trick or discarding an old one? While unlearning theories are appealing because they trigger a natural desire to question one's own knowledge, they become impractical when faced with reality.

Moreover, unlearning isn't always necessary (Klein, 1989; Kuwada, 1998). This means that old and new knowledge may coexist in organizational action and memory. Knowledge such as myths, theories of action, values, beliefs, and methods, represents for the organization what cognitive structures represent for the individual. It filters the environmental information and models organizational behavior (Hedberg, 1981; Sproull, 1981; Lorsch, 1986; Pfeffer, 1981; Starbuck, 2000). While they might be obsolete, these chunks of knowledge contribute to the consistency of action, even if detrimental to its performance. Hence, if such "old knowledge" shall be discarded, then most organizations would lose their consistency, maybe able to learn more, but incapable to act. Organizations would either be paralyzed, or would suffer schizophrenia.

Holding that knowledge determines behavior and that old and new organizational knowledge may coexist sounds paradoxical. However, this paradox can be solved if we bring into discussion the phenomena of knowledge activation and knowledge validity. It will be subsequently argued that old and new knowledge can coexist in organizational memory. However, it is only the one that is both activated and has perceived validity that will eventually influence organizational behavior.

\subsection{Knowledge Validity}

The first two meanings given by American English dictionaries to the word "valid" are: (1) sound; just; well-founded, and (2) producing the desired result; effective: a valid remedy. In the first place, the validity of a piece of knowledge 
is related to its ontological value: is it true or false? From this point of view, validity is normally established by the objective conditions an organizatibl's environment may provide. For instance, the laws of demand in competitive markets state that the decrease of the price of goods will surely augment the amount of consumers' demand for them. This law is generally accepted because economists see it to be true and people may notice and experience it in everyday life.

Unfortunately, things are not always so easily accessible or visible. This usually leads organizational actors away from the realm of objective validity toward perceived validity. This dichotomy is necessary As Bawmard and Starbuck (2005) point out, cognition does not afford dependable basis for learning. Most managers have very erroneous perceptions of both their firms and their business environments (Mezias and Starbuck, 2003). Top managers, for instance, are often rather out of touch with current customers, suppliers, or technologies.

The same discordance between objective and perceived validity may turn the other way around. Management fads, for instance, usually spread this way. The Singer Company embarked on a certain product diversification strategy from 1967 to 1974. Managers noticed that this strategy was successful for other companies (Miller and Friesen 1980). Heavy losses determined by its continuous pursuit didn't manage to shatter the perceived validity of this causal association. Hedberg (1981) called this phenomenton superstitious learning: organizational actors attribute (perceived) validity to a piece of knowledge that is objectively invalid. Such learning usually occurs under conditions of ambiguity, or when the complex interactions bdtween organizations and their environments exceed people's cognitive capacities for mapping, so that faulty inferences are drawn. The clear-cut distinction between perceived and objective validity is very important since it is perceived valid knowledge that shapes and affects organizational behavior outcomes, while objectively valid knowledge, as well as perceived invalid knowledge, may stay unheeded by organizational actors.

All organizational actors try to establish the perceived validity of knowledge against objective data (Daft and Weick, 1984). A part of the organizational knowledge could be validated this way. Unfortunately, however, because a large part of organizational knowledge is socially constructed, the yardstick of objective validity doesn't always exist (Baumard, 1996). In such cases, validity will be defined by its second meaning, that of producing effective outcomes.

\subsection{Validation, Invalidation, and Strategies for Unlearning}

The various strategies for organizational unlearning proposed by previous research are suffused with assumptions concerning the ontology of knowledge. They fall mainly in two categories. The first group (a) focuses on procedures, practices, exchange protocols with the task-environment of the firm, as well 
with certain behavior-outcome associations, rules, norms, and structures. The second group (b) focuses on goal-setting,cultura_beliefs and obsolete causal patterns carried over by the overall orgdnizatioh

Most of these theodies see "first-order khowledge" as a maliz soulrce of learning inertia, whether thlíinentia comes from frozen behavioral routines or organization-wide obsolete belief structures. However, Klein (1989) argued that first-order knowledge mustn't necessarily be unlearned. Its validity can be easily checked because its outcomes_are_immediate and can be interpreted readily. Once it is proved that first-ander knowledge is no longer appropriate, it will be 'bracketed' or marked by invalidity. Therefore obsolete knowledge may last in the organizational memory without bleing deletted and without being subsequently enacted.

Second order knowledge can be understood according to notions such as frames of reference (McCall, 1977), myths and perceptual filters (Hedberg, 1981), theories of action (Arovris and Schön, 1976), and basic assumptions (Kuwada, 1998). This knoulledop plays a major part in organizational life. First, it allows the interpretation df complex environmental and internal configurations due to meta-rules of perception and event expectancies. Thus, it frames the organizational actors' input information, usually without their awareness (Lorsch, 1986). Second, these logics of action will shape decisions and strategies, often in an unconscious manner. The reverse of these is the high complexity of such knowledge (Starbuck, 1983). In addition, the extremely loose coupling between this knowledge and organizational outcomes makes the mechanisms for its validation practically nonexistent. Consequently, secondorder knowledge will seldom change, except under extreme circumstances. For instance, myths will change only by conquest or by ideological contamination (Hedberg, 1981).

Kuwada (1998) studied the process of strategic learning at the corporate level, namely the dynamics of basic assumptions. Basic assumptions serve as devices for sense-making, they determine modes of interpretation, and they underlie interpretation routines. They also condition and shape the design process of corporate-level strategic behavior. Strategic learning could not follow a trial-error process. Basic assumptions are a tacit form of secondorder knowledge, usually entangling strongly held, emotionally loaded beliefs, They describe complex realities that can be hardly invalidated When new assumptions come into competition with old ones, strategic behaviors will be determined alternatively by the old and new assumptions. Thus, they will concomitantly be held in organizational memory, and will be alternatively used until, in the end, the one will be fully validated and the other will eventually fade from organizational memory.

The two preceding examples show that erasing obsolete knowledge is not the only way that allows new organizational knowledge and behavior to develop. Old knowledge may coexist with new knowledge without interference as long as the former is considered invalid. They seldom can both be considered valid, however - a temporary situation which is always accompanied by political conflicts in the organization (Hedberg, 1981). Unlearning and relearning can then hardly be disentangled in such a case. 
Table 1. bjects organizational unlearning in several studies

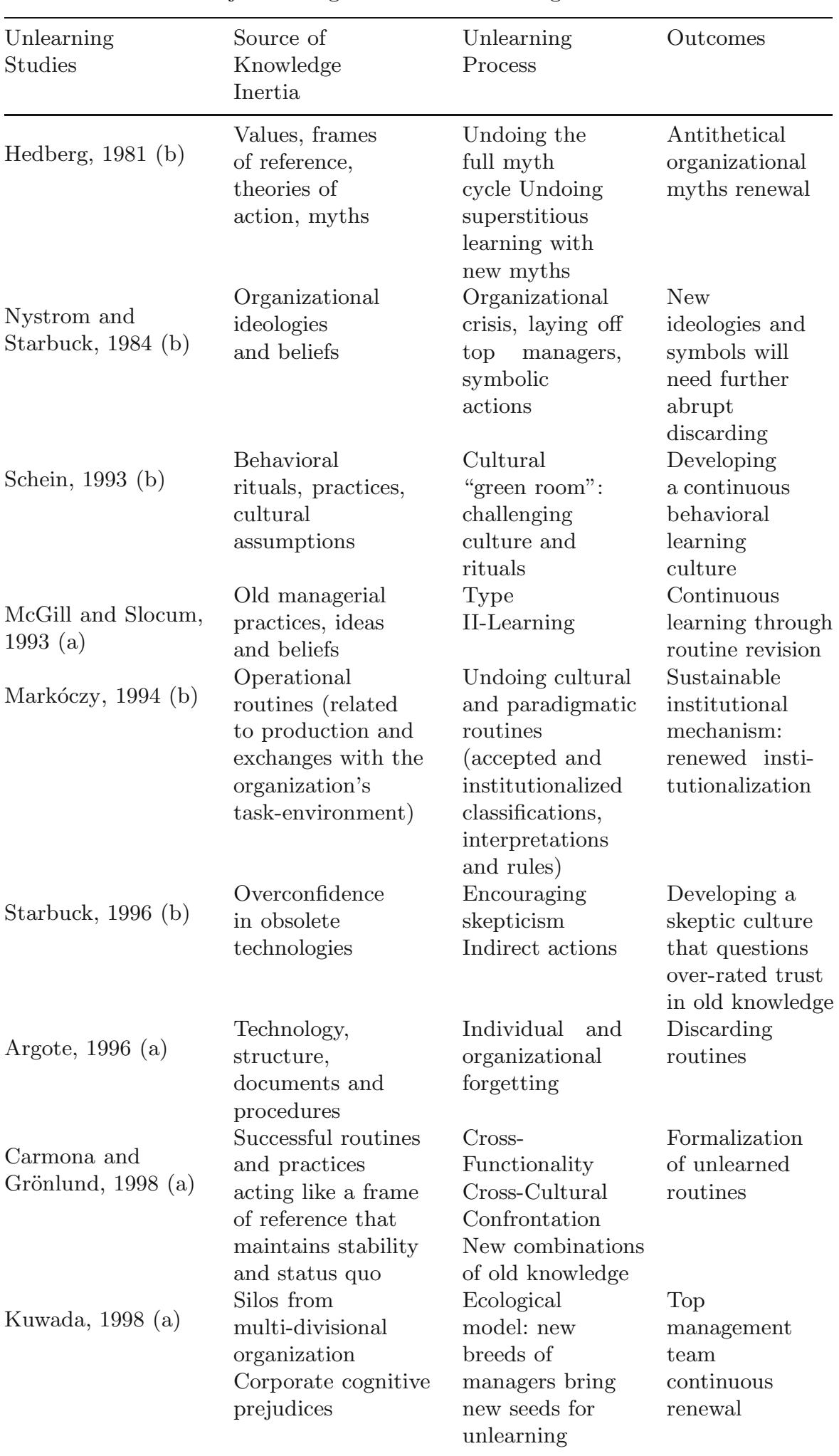




\subsection{Matters of Knowledge Activation $\square$}

Up to this point, it was implicitly assumed that the perceived validity of knowledge would also guarantee its translation into action. It has been shown that individuals, however, do not obey such a law. Argyris' work (1993) suggests that human beings have in their heads more that one design about how to act effectively. Faced with difficult, threatening, or embarrassing issues, individuals' behavior suggests an underlving pattern of cognitions that are labeled "theories-in-use." Neverthbless, nhen questioned about their reasons, the same individuals would describe rationales that are inconsistent with their former actions. These beliefs, undoubted sincert ty, are labeled "espoused theories" (Argyris, 1993). Twb_onclusions are in order. First, though individuals are unaware of their influence, theories-in-use are valid because they are enacted, according to the second meaning of validity. Second, espoused theories also enjoy a perceived validity since people sincerely believe they represent the truth. The two theories are both valid and they deal with the same issues. Yet, only one of them is effectively put into practice.

A similar phenomenon was noticed in organizations. Though firms accumulate important amounts of intellectual capital, a big part of it is never translated into action (Want, 1993). Noticing this knowing-doing gap, Lew Platt, CEO of Hewlett Packard, has justifiably exclaimed: "I wish we knew what we know at HP!" (Pfeffer and Sutton, 1999)

Pfeffer and Sutton (1999) advanced an explanation that seems reasonable for both individual and rganizational cases. The existence of valid knowledge that is not put into practice is explained by the carelessness of firms and individuals concerning tacit knowledge. Organizational knowledge should, in these authors' opinion, be primarily configured in this uncoded, applied form through a process of internalization before it becomes ready to use or active. We will broaden their argument by suggesting that valid knowledge is only activated inside special knowledge repertoires. Only there can it be in a position to influence the organization's strategic behavior.

The existence of such registers flows naturally from the polvmorphism of organizational knowledge (Girod, 1995). For instance, Nonba (1994) highlights the difference between explicit and tacit knowledge. Baumard (1996) differentiates knowledge as individual and collective knowledge according to the number of individuals that share it. The intersection of the two dimensions determines four types of knowledge registers: explicit individual, tacit individual, explicit collective and tacit collective. All the four types of knowledge are present in any organization. Baumard also demonstrated that, faced with ambiguity, organizations react by transferring knowledge from one register to another. In this way, organizations obtain a better mapping of their dynamic environment. They also fan out such knowledge that is fitted to determine appropriate actions for the succeeding phases of ambiguous situations. Kuwada (1998) defines organizational knowledge along another dimension, somewhat similar to the hierarchical 
position of the loci of knowledge. He thus identifies business-level knowledge, mainly present at the business-unit level and characteristic of middlemanagement. A more important type of knowledge is that of corporate-level knowledge, that infuses all the organization and activities performed_Huwada also specifies that it is not until knowledge evolves from business-level to the corporate-level register that it will influence the design process of strategic behavior.

This research allows two important conclusions. First, an organization's knowledge base is heterogeneous. It is made up of different registers where knowledge has particular properties and roles. For instance, tacit knowledge is uncoded but readily usable, while business-level knowledge is grounded, related to very specific business contingencies, but couldn't govern the corporate policies. Second, knowledge flows from one register to another. A piece of knowledge is active only inside the specfic reg ster that governs the corresponding organizational behavior (Kim, 1998). Therefore, the transfer of knowledge from one register to another has either an activating or a deactivating character.

\section{Unlearning and Knowledge Neutralization}

Is the organizational unlearnilng natter artificially developed? Researchers often agree that persons act and learn within the organizational framework and that organizational learning is a result of individual learning (Nicolini and Meznar, 1995; Hedberg, 1981). Or individual unlearning is not as much a matter of discarding knowledge, as it is a matter of reduced response availability. The success of such methods as hypnosis or cortical stimulation, as well as the "spontaneous recovery" of unlearned items, show that nonreproduced items are not necessarily lost for ever (Klein, 1989). On the other hand, organizations can unlearn by eliminating the "hardware" that stores the organizational memory, by firing key personnel, "refreshing" their archives, or by simply loosing their blueprints (Weick, 1999).

Miller (1978) signaled that in most debated, dianhetrically opposed adversaries may be each correct-but in different contexts. Actually, unlearning theories have mostly been developed for organizations facing crisis when time is short because survival is at stake. Quick invalidation is difficult, especially for second-order knowledge. Hence, more radical measures as the exclusion of organizational knowledge - unlearning - are justified. On the other hand, organizations that are not faced with dangerous environmental deadlines may forsake the unlearning/relearning cycle for a piecemeal incremental learning approach. Hence, people in organizations might well be aware of the obsolete nature of part of their knowledge, and strategically chose to put it aside, either for political motives, or as to avoid liability in a potential failure (Baumard and Starbuck, 2005). Prior knowledge cannot influence organizational behavior once it is excluded from organizational memory. As 
Baumard and Starbuck (2005) observed in their fourteen case studies of small and large organizational failures, unlearning is often prevented by erasing or rewriting history, moving people around, or re-qualifying painful experiences as healthy and most needed experimentations.

Hence, people and organizations alike fail to unlearn, because they have put aside the repository of knowledge that contains the ground for unlearning. While accepting the existence of these knowledge repositories, unlearning could still be achieved by two other ways - either through rivaling enactment or through knowledge inactivation. This is what we label as "knowledge neutralization." Neutralization refers to purposefully destroying a peculiar or opposite disposition inherited or embedded in a knowledge body. For example, core beliefs of an organization create a "feeling of knowing" and encourage the pursuit of the application of obsolete knowledge. Neutralization is the process by which managers may counteract the effect of these core beliefs and obsolete knowledge on their decision making.

Learning mechapisms emerged in historical contexts, and bear with the the prejudices, jurlsdictions and founding flaws of their first design. For instance, in large disasters, many jurisdictions and organizational bodies have to reconcile years of separate learning into one consistent and coordinated ephemeral organization. Many discrepancies in larose-scale emeroencies come from the "archeology of learwing" of the different organizations cooperating. Different layers of learning habits become heterogeneous sediments in each organization. They impede new learning by preventing new knowledge to settle or being considered. We thus define "archeology of learning" as the accumulation at length of layers of learning experiences, habits and systems that produce the current learning system or mechanism of an organization. In their study of the NASA Challenger shuttle disaster, Starbuck \& Milliken (1988) point out the critical role of "perceptual filtering" in the event of unseen disasters. In time, organizations tend to reinforce their core beliefs, by discarding small failures as just a reinforcement of commitment to their beliefs, and large failures as external and historical exceptions (Baumard and Starbuck, 2005). Reward systems and incentives in organizations indeed favor parochialism, as people are rewarded for improving knowledge in their field, in respect of their attributed jurisdiction. Individualistic modern cultures, in both Europe and the U.S., also favor the expertise of the few, over the value of knowledge sharing. As a result, most organizations, public and private, encourage by design the creation of "learning silos" within their walls.

A major consequence of these "learning silos" resides in the tunnel visions that jurisdictional entities develop over time. In times of normal operating conditions, adjustments between the different bodies in charge of a systemic environment are accomplished through "fine tuning." Exceptions are tolerated, and for example, a passage made through a levee for train railroad will be accommodated, as we saw in the 2005 hurricane Katrina disaster in New Orleans, as far as it does not challenge the core beliefs of the respective concerned groups. Over time, all these small "adjustments," "corner cuts," 
create spaces where learning do not have any ownership. While a cut in the core belief has been accepted, the concerned zones become a "no challenge" zone for the respective learning systems. When large-scale disasters arise, the history of the "corner cuts" is lost. Fine-tuning that might create a serious threat to critical infrastructure has escaped both the learning systems and the memory of current actors. The "structural holes" in infrastructure learning are both the results of the sediments of old obsolete learning systems and on-going fine-tuning that create "no care" zones in jurisdictional systems.

Hence, organizations are struggling with an evolutionary neutralization of their knowledge base, while being over-influenced by core beliefs, and mostly incapable of purposefully choosing which knowledge should be neutralized, and which should be put forward. For instance, in the Katrina's disaster response, the Army Corps of Engineers, in charge of levees maintenance, had little influence over urbanization that is outside of their legitimate jurisdiction. When levees are erected they require a sufficiently empty ground around them for further elevation. The City of New Orleans overlooked this requisite, and encouraged homeowners to build near to these levees. While levees and flood control are outside of the City's jurisdiction, expertise and learning curves on this specific matter have been naturally neutralized over time. As this example shows, neutralization of previous learning can be either desired or overlooked.

Several types of knowledge are at play in this interaction between neutralized learning over time and the upcoming of new knowledge. The subsequent section will provde details concerning the processes at work for each identified type of knowledge neutralization. Attention will be paid also to the circumstances when they apply most and to the management literature that illustrates them.

\subsection{Knowledge Neutralization Through Rivaling Enforced Enactment}

The invalidation of second-order knowledge is usually considered to be a difficult undertaking. First, this knowledge is often invibiblot organizational actors. Few individuals are aware of their beliefs, values or assumptions (Lorsch, 1986). Second, it is very loosely coupleld to organizational performance. On the one hand, second-order knowledge doesn't wholly determine organizational actions. It rather shapes them in interaction with other types of knowledge. For instance, decision-makers ponder environmental information, norms, outside opinions, and their own beliefs and values before enacting a specific course of action. On the other hand, performance may be time lagged. It can also correspond to the composed result of cumulated, distinctive organizational actions (Spender, 1996). Thus, a specific piece of second-order knowledge becomes very loosely coupled to performance indicators (Glassman, 1973). Trial-error invalidation cannot function. Negative outcomes will be merely accounted for as accidental, historical or temporary phenomena (Baumard and Starbuck, 2005). 
For instance, in the response to large scale disasters, the different involved organizations act upon their own peculiar second-order knowledge. The Army Corps of Engineers will work on preventing water to overflow the levees, for it is their core belief that the magnitude of flood levels has been identified in the past, and is within a predictable range. City planners on the other hand act upon their own secondary knowledge and will answer to urban growth by eliminating the marsh that surrounds New Orleans. It has been forgotten, however, that these marshes have been taken into account in calculating the level of levees by the French engineers who created the water barrier around "la Nouvelle Orleans" two centuries earlier. The Army Corps is evaluated on the maintenance of the current levees, not on challenging the systemic organization of the whole region. Meanwhile, City Planners are evaluated by their good handling of rapid growth, not on making sure that the city is surrounded by levees. Both organizations act on their own rights, and both are legitimate in doing so. As they test their beliefs against their own jurisdictional experimental grounds, these beliefs always come back validated. Hence, even trials and errors are likely to reaffirm the core beliefs (Baumard \& Starbuck, 2005).

When trial-error mechanisms are futile, knowledge may be invalidated according to a principle of frequency. Postman and Underwood's (1973) study of the dynamics of association supports this view at the individual level. In their research, individuals were first conditioned to expect that Lan avent B would follow an event A. Experimenters subsequently substituted event B with event $\mathrm{C}$. The repeated occurrence of the $\mathrm{A} \rightarrow \mathrm{C}$ association began at some point to replace the old association $\mathrm{A} \rightarrow \mathrm{B}$. The latter was gradually transferred to a less accessible area of the individuals' memories. Humans' associative memories are thus updated through dynamic reallocations based on the frequencies of the observed relationships.

Repetition also influences the validity of knowledge at the organizational level. Top managers often hold different world-views, act according to different paradigms, and want to guide corporate strategic actions following their personal beliefs. Managerial beliefs surfacing at the organizational level usually turn into basic assumptions. Kuwada (1998) argues that the selection mechanism of these assumptions depends on environmental support and repetition. Unused basic assumptions loose perceived validity. The process also works the other way round: the lessening of perceived validity decreases the use of respective basic assumptions. A vicious circle sets in: lesser use, lesser perceived validity, and lesser use. These considerations lead to a first, frequency-based, principle of neutralization. The more frequent the enactment of new, rivaling knowledge, the lesser the perceived validity of old knowledge, and vice versa. In time, old knowledge generates less behavioral outcomes than new knowledge.

Neutralization through rivaling enforced enactment evolves through long periods of time. No direct actions can be undertaken to suddenly shatter deeply embedded beliefs or worldviews held by numerous organizational actors. Nevertheless, neutralization can be accomplished by initiating the 
circle of invalidation through domination. One way to start this dynamics is by doubting that the beliefs, values, knowledge, information, abilities, and skills that are held are necessarily true or valid. As Weick (1999) pointed out, the opposite of crediting is doubt, rather than disbelief. Therefore, doubt is likely to slightly reduce the perceived validity of organizational knowledge, thus putting in motion the neutralization circle.

One way to insert doubt in organizational life is by altering the organizational culture in order to accommodate Starbuck's (1996) following eight viewpoints:

1. "It isn't good enough" - dissatisfaction is probably the prevalent reason for doubting current knowledge;

2. "It's only an experiment" - make people feel like experimenters, they will probably alter their beliefs and methods and look for new insights;

3. "Surprises should be question marks" - both pleasant and unpleasant surprises may engender doubt;

4. "All dissents and warnings have some validity" - if there is dissent, it might be well founded, therefone managers should not overlook it;

5. "Collaborators who disagree are both right" - qualified observers always have foundations in some sort of truth, even if they disagree;

6. "What does a stranger think strange?"-insiders need outside opinions in correction to their own. Outsiders may provide startling insights that cannot be generated inside of the organizational setting;

7. "All causal arrows have two heads" — organizational actors are invited to dialectical reasoning in order to challenge their own tacit assumptions;

8. "The converse of every proposition is equally valid" - dialectical reasoning should not be confined only to causal insights, but to all kind of organizational knowledge.

Lorsch (1986) proposed an alternative method that could be used in order to impel the change of basic beliefs in organizations. The main argument is that invisible beliefs are difficult to fight, especially when they generate strong emotional commitment. An audit is recommended that should identify beliefs shared by top managers. The results ought to be made visible, explained, hung on the walls. If managers become aware of their beliefs, they are less likely to be blinded by them and are apt to understand and to deal more rapidly in the face of change, retaining beliefs that are reasonably valid and gradually dropping out those that are not.

The two previous examples show that knowledge neutralization through rivaling enactment is fundamentally related to cultural dynamics. Organizational culture should be modified or made visible in order to foster doubt and to set in action the creation and enactment of new knowledge. Such process is inevitably time consuming. Furthermore, in order to influence knowledge neutralization, one should be able to alter an organization's culture. Therefore, the actors that are more likely to trigger this process are charismatic leaders or external auditors. Large, integrated, planned, 
organizational changes are the only instances of purposive organizational change where knowledge neutralization through rivaling enactment could be intently triggered and performed.

\subsection{Knowledge Neutralization Through Knowledge Inactivation}

Whenever managers don't have the means or time to influence the perceived validity of organizational knowledge, they should try to neutralize it by an inactivation process. According to our previous argument,knowledge can influence organizational behavior only once it has been cbnfigured inside specific knowledge registers. Whereas Pfeffer and Sutton (1999) discussed the process of knowledge activation, it can be conjectured that valid knowledge may also be inactivated, provided that one caln forct it to move to an unfavorable register (Spender and Baumard, 1995). Therefore, knowledge inactivation may be defined as neutralization based on interregister knowledge dynamics. Once transferred into an unfavorable register, organizational knowledge is mable to influence antient target-behaviors and becomes inactivated.

Although this issue has not been discussed yet under these terms, the literature provides case studies that could be interpreted as knowledge inactivation. The research performed by Carmona and Grönlund (1998) on two car manufacturer subsidiaries is such an example. The main purpose of the study was to develop insights about the learning and forgetting processes according to an experience curve paradigm (Argote, 1996). Hereby we suggest that the process called forgetfulness by the two authors may be interpreted as an instance of knowledge inactivation.

The event under study was the setup of task forces at the shop-floor level (Carmona and Grönlund 1998). Their main objective was to fluidize the production flows constrained by several bottleneck areas, to increase capacity and production compliance, and to improve quality and working conditions. Teams were formed by a diversified range of employees such as operators and middle managers - an industrial engineer, maintenance engineer, manufacturing engineer, maintenance foreman, and front line supervisor.

During a first phase, the trajectories followed by the working teams of the two firms were quite similar. Members drew lists of problems. They also gathered information that was fully independent from the one provided by the central budgeting systems. Items were discussed in order to ameliorate product quality, production schedule compliance, and the consumption of maintenance services. Solutions were proposed and implemented. The researchers interpreted the subsequent overall increase of production outcome as a proof of organizational learning.

The second phase brought a differentiation between the trajectories of the two task forces. The Swedish factory acknowledged the performances of their working team and decided to offer support by providing it with formal status 
and by rewarding the efforts of the participants according to a comprehensive reward system. The production level obtained was thus further maintained and continued to progress slowly. In the Spanish subsidiary the situation was largely different. The lack of formal status was cruelly experienced when the task force had to deal with problems outside of its area of influence. The efforts of team members were also poorly rewarded. At length, individuals returned to their previous practices, disappointed with the manufacturer's inattention to their efforts. Production levels fell rapidly to the levels existing before the setup of the informal team.

Carmona and Grönlund (1998) interpret the phenomena, respectively, as knowledge maintenance and as a prodcess forgetting. They argued that the inclusion of the informal Swedish team in the organization chart acted as a shield against the dissipation of new knowledge creatdd by the working teams. The absence of such a shield led to the loss of this knowledge in the Spanish plant.

The evolution of the two cases may remind one of the definition of communities of practice: "Within communities-of-practice, people share tacit knowledge and through dialogue bring this to the surface; they exchange ideas about work practice and experiment with new methods and ideas; they engage in discussions which affirm or modify theories in use; they innovate new problem-solving routines and simultaneously manage and repair the social context" (Hendry, 1996). Though one might argue that the wdrking teams never became communities-of-practice since they reunited people with very different expertise (Wenger and Snyder, 2000), their trajectories and modus operandi demonstrate that they did engage in the creation of tacit, collective knowledge that bolstered production levels. Such knowledge only exists and acts as long as it is supported by a special social context based on frequent interaction and exchanges among individuals. While such social structures were sustained and confirmed in the Swedish plant, they were submitted to a rapid process of erosion in the Spanish one. Though in the latter case the structures fell apart and performance was lost due to knowledge inactivation, it is reasonable to suppose that reinstating those structures after a short while would have reactivated knowledge that Carmona and Grönlund (1998) considered lost or forgotten. Thus, one may suppose that knowledge did not disappear, nor was it forgotten, but it passed in a first phase from the tacit, collective register to the tacit individual one, where it became inactive in the absence of interaction.

Purposive knowledge inactivation may prove to be a difficult endeavor. First, managers should identify detrimental knowledge and the registers where it is configured (e.g., tacit, collective knowledge). Second, they will have to figure out the registers where this knowledge may be inactivated (e.g., explicit, individual knowledge). Third, managers should find a way to transfer this knowledge from one register to another. Few scientists suggested controls for such knowledge dynamics, save for Osterloh and Frey's (2000) finding that motivational levers might influence knowledge transfers. However, once these 

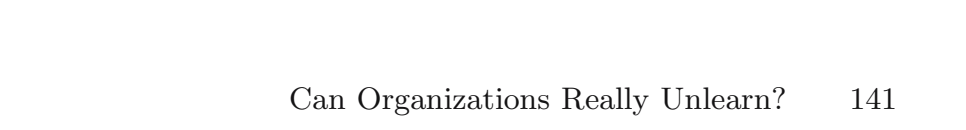

elements are uncovered, knowledge inactivation may be attained in medium term organizational changes.

\subsection{Neutralizing Knowledge Through Unlearning}

Although the last in the order of our discussion, this type of knowledge neutralization seems at the same time the easiest, the fastest, and the most common. It is performed either through the bysical brth cognitive expulsion of knowledge from the organizational memory (Starbuck, 1989; Hedberg, et al. 1976; Nystrom and Starbuck, 1984; McGill and Slocum, 1993).

Knowledge management has always been fascinated with the metaphor of thinking machines, an image that usually has a limited utility (Starbuck, 2000). Nevertheless, this metaphor can be used in this case because organizational knowledge, just like computer stored and treated information, cannot exist in the absence of a storage device. Such a role is performed in computers by ROM and RAM memories: hard disks, floppies, CDs and the like, while organizational knowledge is stored in the organization's technology, its structure, documents, standard operating procedures, and most especially its members (Argote, 1996). Knowledge can depreciate if any of these elements are lost or affected: individuals who leave the organization, technologies that become inaccessible or difficult to use, organizational records and routines that are lost or become difficult to access. A manager that identifies the undesirable knowledge can simply get rid of it by expelling its support. Therefore, knowledge neutralization through unlearning is based on a principle of exclusion. It is the fastest alternative for neutralization. as organizations eliminate undesired behaviors by discandlng the underlying obsolete knowledge.

A very important part of organizational knowledge is shared by the organizational members. Driving away obsolete knowledge by eliminating its support becomes unrealistic. Managers can rarely afford to fire all the people that share it without hindering the good functioning of organizations. It has been noticed, though, that interesting effects are at work while firing the promoters of such shared knowledge. The dismissal of top-rhanagement teams for instance, besides the elimination of individual knowledge and experience, also has a symbolic dimension. People tend to associate the latter's departure with the invalidity of the old methods, beliefs, and strategies, of which they were fierce promoters (Starbuck, 1989; Hedberg, et al. 1976). Such an event will thus accomplish two tasks: first the elimination of top-managers' knowledge, and second the invalidation of shared second-order knowledge such as beliefs, methods, and strategies that they have been supporting or promoting.

As argued above, such knowledge is not unambiguously related to performance indicators and might be invested with political stakes. In their study of a large divisional telecom firm, Baumard and Starbuck (2005) showed that firms learn sometimes surprisingly little from failures: "managers find 
it easy to explain both large and small failures as having idiosyncratic or exogenous causes that no one could have foreseen, and to rationalize their personal actions in terms of their firm's core beliefs [...] The learning that should follow faidre often does not occur, and when it does occur, it often teaches the wrong lesson." (p. 295). Nevertheless, cognitive expulsion can be performed on first-order knowledge, such as programs or standard operating procedures that are tightly coupled with an immediate outcome. For instance, a manager can neutralize undesired practices by explicitly and formally banishing them through the scripts assigned to the respective organizational roles. This method is largely present for instance on high reliability organizations such as nuclear aircraft carriers or nuclear plants, where destructive mal-practices are explicitly forbidden. Though such a method is readily available, specialists may have to spend a lot of time until they are able to identify negative first-order knowledge! (Starbuck, 1996). For instance, people in organizations tend to confuse first and second order knowledge. What are merely behavioral habits (first order) are often taken fo granted as rightful molds and paradigms. onversely, unnoticed, unacknowledged beliefs and ideologies (second order) generate automatic behavioral responses that people tend to see as rational actions (e.g., rational myths).

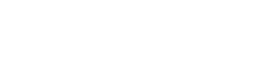

\section{Discussion and Conclusion}

To this day, organizational literature has attributed two main roles to the unlearning process. On the one hand, unlearning was considered an unavoidable precedent for effective organizational learning (Starbuck, 1989; Nystrom and Starbuck, 1984; Hedberg, et al. 1976). Even if such a priori didn't gather widespread recognition, it is generally acknowledged that discarding obsolete knowledge makes way for new knowledge, thus fostering the relearning process. On the other hand, unlearning was considered a trigger for new learning (Hedberg, 1981). The removal of knowledge would signal change to disoriented organizational actors. Unfortunately, such triggering couldn't be controlled. According to this paradigm, unlearning is mainly induced by major adverse environmental shifts.

This article generalizes organizational unlearning toward the notion of knowledge neutralization. Unlike the former concept, knowledge neutralization cannot pretend to have an inevitable role in organizational learning. It merely represents a group of phenomena that results in the disappearance of obsolete behavior through knowledge manipulation. Nevertheless, it can be used as a powerful support tool in organizational change. One of its main roles is to help organizations get rid of detrimental behavior. Although precedence is no longer an issue, the unavailability of past behavior makes it possible for employees to embark on a quest for new knowledge and to experiment, thus fostering new learning. 
It has been argued that knowledge neutralization is a controllable process. Unlike the classical point of view on unlearning, agents of change may trigger particular instances of knowledge neutralization, according to the resources and time available. For instance, organizational designers could embed Starbuck's (1996) eight viewpoints in a new, flexible, competitive organization. If shared obsolete knowledge results in reiterating bad results or dissent, managers could try to identify its locus and inactivate it through a transfer to an unfavorable knowledge register. Finally, time shortages may justify a manager's actions directed to organizational unlearning. Eliminating knowledge this way may be painful, but it is sometimes a better alternative than slow erosion and eventual bankruptcy.

In conclusion, managers should use knowledge neutralization techniques selectively, according to organizational and environmental contingencies in terms of resources and type of targeted knowledge. By eliminating obsolete behavior, knowledge neutralization becomes an adaptive tool for facilitating and accelerating organizational change.

Recent developments in organizational science outline the importance of knowledge creation. Many scientists are concerned with learning organizations: fewer refer to unlearning organizations. Obviously, the gathering and activation of knowledge are no trivial actions. They are consuming of time, resources, and attention. Hopefully, obsolete knowledge or behavior will, at length, fade away and make way for new knowledge or behaviors. But in a world where resources and time for change are short, such lingering is likely to promote ideologies rather than produce real unlearning. Organizations may be likely to unlearn, if they start by unlearning the way they currently unlearn.

\section{References}

Argote, L. (1996). Organizational learning curves: persistence, transfer and turnover. International Journal of Technology Management, Special Issue on Unlearning and learning for Technological Innovation, 11 (7/8), 759-769.

Argyris, C. (1993). Education for leading-learning. Organizational Dynamics, 21 (3) $5-17$.

Argyris, C., \& D. A. Schön. (1976). Organizational learning:A theory of action perspective. Reading, Mass: Addison-Wesley.

Baumard, P. (1996). Organisations déconcertées. La gestion stratégique de la connaissance. Paris: Masson.

Baumard, P. (1999). Tacit knowledgein organizations. London: Sage.

Baumard, P., \& Starbuck, W.H. (2005). Learningfrom failures: Why it may not happen. Long Range Planning. 38, 281-298.

Carmona S., \& Grönlund, A. (1998). Learning from forgetting: An experiential study of two European car manufacturers. Management Learning, 29 (1) 21-38.

Daft, R. L., \& Weick, K. E. (1984). Toward a model of organizations as interpretation systems. Academy of Management Review, 9 (2), 284-295. 
Fiol, C. M., \& Lyles, M. A. (1985). Organizational learning. Academy of Management Review, 10 (4) 803-813.

Firsirotu, M. E. (1985). Strategic Turnaround as Cultural Revolution: The Case of Canadian National Express. Unpublished doctoral dissertation, McGill University.

Girod, M. (1995). La mémoire organisationnelle. Revue Française de Gestion. 105, 30-42.

Glassman, R. B. (1973). Persistence and loose coupling in living systems. Behavioral Science, 18, 83-98.

Hatch, M. J. (1993). The dynamics of organizational culture. Academy of Management Review, 18(4), 657-693.

Hedberg, B. L. T. (1981). How organizations learn and unlearn. In P.C. Nystrom \& W.H. Starbuck (Eds.), Handbook of Organizational Design (1, 3-27). New York: Oxford University Press.

Hedberg, B. L. T., Nystrom, P. C., \& Starbuck, W. H. (1976). Camping on seesaws: Prescriptions for a self-designing organization. Administrative Science Quarterly, 21, 41-65.

Hendry, C. (1996). Understanding and creating whole organizational change through learning theory. Human Relations. 49 (5), 621-641.

Kilmann, R. H. (1989). A completely integrated program for organizational change. In A.M. Mohrman Jr., S.A. Mohrman, G. E. Ledford Jr., T. G. Cummings, E. E. Lawler III, \& Associates (Eds.), Large-Scale Organizational Change (pp. 200-228). San Francisco: Jossey-Bass.

Kilmann, R. H., Saxton, M. J., \& Serpa, R. (1986). Issues in understanding and changing culture. California Management Review, 28 (2), 87-94.

Kim, L. (1998). Crisis construction and organizational learning: Capability building in catching-up at Hyundai Motor. Organizational Science, 9 (4), 506-521.

Klein, J. I. (1989). Parenthetic learning in organizations: Toward the unlearning of the unlearning model. Journal of Management Studies, 26 (3), 291-309.

Kuwada, K. (1998). Strategic learning: The continuous side of discontinuous strategic change. Organization Science, 9 (6), 719-736.

Lei, D., J. W. Slocum, J. W., \& Pitts, R. A. (1999). Designing organizations for competitive advantage: The power of unlearning and learning. Organizational Dynamics, 27 (3), 24-38.

Lorsch, J. W. (1986). Managing culture: The invisible barrier to strategic change. California Management Review, 28 (2), 95-109.

Markóczy, L. (1994). Modes of organizational learning: Institutional change and Hungarian joint ventures. International Studies of Management and Organization, 24 (4), 5-30.

McCall, M. W. (1977). Making sense with nonsense: Helping frames of reference clash. In P.C.Nystrom. \& W.H. Starbuck (Eds.), Prescriptive models of organizations (pp. 111-123). Amsterdam: North-Holland Publishing.

McGill, M. E., \& Slocum, J. W. (1993). Unlearning the organization. Organizational Dynamics, 22 (2), 67-79.

Meyer, A. D. (1982). Adapting to environmental jolts. Administrative Science Quarterly, 27, 515-537.

Mezias, J.M., \& Starbuck, W.H. (2003). Studying the accuracy of managers' perceptions: A research odyssey. British Journal of Management, 14, 3-17. 
Miller D., \& Friesen, P. H. (1980). Momentum and revolution in organizational adaptation. Academy of Management Journal, 23 (4), 591-614.

Miller, D. (1978). The role of multivariate "Q-techniques" in the study of organizations. Academy of Management Review, 3, 515-531.

Nicolini, D., \& Meznar, M. B. (1995). The social construction of organizational learning: Conceptual and practical issues in the field. Human Relations, 48 (7), $727-746$

Nonaka, I. (1994). A dynamic theory of organizational knowledge creation. Organization Science, 5 (1), 14-37.

Nystrom, P. C., \& Starbuck, W. H. (1984). To avoid organizational crises, unlearn. Organizational Dynamics, 12 (4), 53-65.

Osterloh, M., \& Frey, B. S. (2000). Motivation, knowledge transfer, and organizational forms. Organization Science, 11 (5), 538-550.

Pfeffer, J. (1981). Management as symbolic action: The creation and maintenance of organizational paradigms. Research in Organizational Behavior, 3, 1-52.

Pfeffer, J., \& Sutton, R. I. (1999). Knowing "what" to do is not enough: Turning knowledge into action. California Management Review, 42 (1), 83-108.

Postman, L., \& Underwood, B. J. (1973). Critical issues in interference theory. Memory and Cognition, 1, 19-40.

Schein, E. H. (1993). How can organizations learn faster? The challenge of entering the Green Room. Sloan Management Review, 34 (2), 85-92.

Schieman, W. A. (1992). Organizational change: Lessons from a turnaround. Management Review, 81 (4), 34-37.

Slatter, S. St.P. (1984). The impact of crisis on managerial behavior. Business Horizons, $27(3), 65-68$.

Spender, J.-C. (1996). Organizational knowledge, learning and memory: three concepts in search of a theory. Journal of Organizational Change Management, $9(1), 63-78$.

Spender, J.-C., \& Baumard, P. (1995). Turning troubled firms around: Case evidence for a Penrosian account of strategic recovery. The Academy of Management Conference. Vancouver.

Sproull, L. S. (1981). Beliefs in organizations. In P.C. Nystrom \& W.H. Starbuck (Eds.), Handbook of Organizational Design (2, 203-224). New York: Oxford University Press.

Starbuck, W. H., \& Milliken, F. J. (1988). Challenger: Changing the odds until something breaks. Journal of Management Studie, 25, 319-340.

Starbuck W.H., \& Laudon, K. (1996). Organizational information and knowledge. In M. Warner (Ed.), International Encyclopedia of Business and Management (pp. 3923-3933). London: Routledge/Thompson Business Press.

Starbuck, W. H. (1983). Organizations as action generators. American Sociological Review, 48, 91-102.

Starbuck, W. H. (1989). Why organizations run into crises ... and sometimes survive them. In K.C. Laudon \& J. Turner (Eds.), Information Technology and Management Strategy (pp. 11-33). Upper Saddle River, NJ: Prentice Hall.

Starbuck, W. H. (1996). Unlearning ineffective or obsolete technologies. International Journal of Technology Management, Special Issue on Unlearning and learningfor Technological Innovation, 11 (7-8), 725-737. 
Starbuck, W. H. (2000). Is Janus the god of understanding? In T. Lant \& Z. Shapira (Eds.), Managerial and Organizational Cognition (pp. 351-365). Mahwah, NJ: Lawrence Erlbaum Associates.

Villinger, R. (1996). Post-acquisition managerial learning in central east Europe. Organization Studies, 17 (2), 181-206. Want, J. E. (1993). Managing radical change. The Journal of Business Strategy, 14 (3), 21-28.

Weick, K. E. (1993). The collapse of sensemaking in organizations: The Mann Gulch disaster. Administrative Science Quarterly, 38, 628-652.

Weick, K. E. (1999). The socialpsychology of organizing. New York: McGraw-Hill.

Wenger, E. C. \& Snyder, W. M. (2000). Communities of practice: The organizational frontier. Harvard Business Review, 78 (1), 139-145. 